\section{El ejercicio del derecho a la educación y la comunicación en la formación universitaria a partir de una propuesta de extensión}

\author{
Aixa Boeykens \\ Directora del Proyecto Prácticas \\ Integrales y Territorio de la Secretaría \\ de Extensión Universitaria y Cultura \\ Universidad Nacional de Entre Ríos, \\ Argentina. \\ aixaboeykens@hotmail.com
}

Integración de la docencia y la extensión /

Intervenciones

RECEPCIÓN: 24/06/16

ACEPTACIÓN FINAL: 10/10/16

\section{Resumen}

El Proyecto de Curricularización de la Extensión "Periodismo en la Universidad y la Escuela Secundaria desarrolla producciones comunicacionales entre estudiantes y docentes de ambos niveles educativos. Este proceso de trabajo sitúa a la extensión como una instancia de diálogos y aprendizajes entre actores e instituciones. Se producen contenidos periodísticos desde una perspectiva que considera a la comunicación como un derecho y a los medios como un servicio público. A partir de 2016 y durante 2017 investigaremos qué incidencias tiene la formación en extensión en los estudiantes del ciclo medio y superior. Este trabajo se lleva adelante en el Taller de Producción Periodística de la Licenciatura en Comunicación Social de la Facultad de Ciencias de la Educación de la Universidad Nacional de Entre Ríos en conjunto con la cátedra Sociología de la Educación.

\section{Resumo}

O Projeto de Curricularização da Extensão "Jornalismo na Universidade e no Ensino Médio" desenvolve produções comunicacionais entre alunos e professores de ambos níveis de ensino. Este processo coloca a extensão como uma instância de diálogo e aprendizado entre atores e instituições. Os conteúdos jornalísticos são produzidos desde uma perspectiva que considera a comunicação como um direito e aos meios de comunicação como um serviço público. A partir de 2016 e durante 2017, pesquisaremos que incidências têm a formação en extensão dos estudantes do Ensino médio e do nível universitario. Este trabalho é realizado na oficina de Produção Jornalística do Bacharelado em Comunicação Social da Faculdade de Ciências da Educação da Universidad Nacional de Entre Ríos conjuntamente com a cadeira de Sociologia da Educação.
Palabras-clave

- Curricularización

- Extensión

- Periodismo

- Universidad

- Enseñanza secundaria
Palavras-chave

- Curricularização

- Extensão

- Jornalismo

- Universidade

- Ensino Médio
Para citación de este artículo

Boeykens, A. (2016). El ejercicio del derecho a la educación y la comunicación en la formación universitaria a partir de una propuesta de extensión. En Revista $+E$ versión digital, (6), pp. 416-421. Santa Fe, Argentina: Ediciones UNL. 


\section{Presentación}

Compartir la experiencia de extensión que venimos desarrollando nos convoca a dar cuenta de algunas perspectivas desde las que nos situamos como actores que integramos un colectivo que es la universidad. Si coincidimos con que esta es un bien público y social, un derecho humano universal y una responsabilidad de los Estados, tal como expresó en 2008 la Declaración Final de la Conferencia Regional de Educación Superior en América Latina (IESALC) de la Organización de las Naciones Unidades para la Educación, la Ciencia y la Cultura (UNESCO), entonces la educación superior es parte del bien común, del conjunto de los hombres y, por lo tanto un derecho que permite nombrar una dignidad deseable de lo humano y una responsabilidad de los Estados (Rinesi, 2015). Aquella universidad cerrada que formaba elites se reformula en nuestro siglo como un espacio del sistema educativo que - junto con los otros niveles: inicial, primario y secundario- son parte de la esfera pública, del bien común. Considerado así, nuestro modo de pensar la vida colectiva demanda interrogarnos cómo nos desenvolvemos y qué estrategias ponemos en juego para garantizar estos derechos y, también, las obligaciones que devienen de éstos. En Argentina, nuestro sistema educativo se asienta sobre el orgullo y prestigio de tener una universidad pública de acceso gratuito que reconoce sus fundamentos en lo que estableció la Reforma Universitaria de 1918: autonomía universitaria; cogobierno; acceso a la docencia por concursos de oposición; periodicidad de las cátedras; desarrollo de investigación y extensión.

Ahora bien, es necesario que pensemos cómo esos principios fundamentales de nuestra universidad se materializan, se tornan en acciones concretas que permiten a estos sujetos estudiantes que se apropien de este espacio que es un bien social que les pertenece. La obligatoriedad del sistema secundario que comenzó a regir en 2006 con la Ley de Educación Nacional n² 26206 nos interpela como universitarios puesto que hay otros sectores sociales que ahora acceden a ella y que anteriormente lo hacían en menor medida. Es también una obligación de nosotros, los profesores universitarios, interrogarnos por los modos con que enseñamos y las políticas educativas para ser capaces de que se garanticen los derechos y obligaciones de poder estudiar y de lograr brindar la más alta calidad para todos.

En este marco, la extensión universitaria se plantea como una parte del proceso del derecho a la educación. Si las prácticas de décadas anteriores consideraban a la extensión en la universidad como el momento en que quienes tenían sus saberes expertos divulgaban o "extendían" sus competencias con aquellos que no; el modo de trabajar hoy supone un "entre". Esto es: un diálogo entre actores universitarios y organizaciones o instituciones que buscan un trabajo conjunto en el que efectivamente los universitarios aportarán sus conocimientos pero a través de un marco de actividades de escucha de necesidades, diálogo, acciones y aprendizaje recíproco.

En el caso del proyecto de extensión que llevamos adelante, nos preguntamos de qué modo los actores que ocupamos el espacio público universitario y secundario nos desenvolvemos en nuestros roles de modo de contribuir a que los estudiantes de ambos niveles puedan formarse, desenvolverse en sus trayectorias académicas con calidad educativa y comunicar en el espacio público a través de un paradigma que considera a la comunicación un bien social. 
Conviene aclarar en este punto que el derecho a la expresión y a la comunicación es pensado como un derecho individual pero también, y fundamentalmente, colectivo. No es suficiente pensar que el derecho a la libertad de expresión se consigue asegurando la libertad de cualquier ciudadano de hablar y expresarse en un espacio público sin que nadie lo reprima o cercene su voz. Hablar del ejercicio de estos derechos supone recibir información diversa, plural y de calidad pero igualmente generar las condiciones necesarias para poder producirlas. A la vez, implica conocer que la información es una construcción y que además de los medios masivos hay otros medios de comunicación que proponen otro tipo de contenido y estética. Como expresa Rinesi:

"No basta con la idea negativa de que nadie nos pegue por decir lo que pensamos. Debe haber una idea positiva de que el Estado, garante de ese derecho, nos genere las condiciones materiales, tecnológicas, etcétera, para poder hablar tan fuerte como cualquiera. $Y$ nos genere las condiciones para que no haya uno grandote que grite tanto que nuestra voz no se oiga. Entonces el derecho que la terminología clásica llamó "libertad de expresión" es un derecho más complejo de lo que podíamos pensarlo en el siglo XIX cuando bastaba llevar un banquito a la esquina de alguna plaza y decir así lo que se nos ocurriera. Pero además, el derecho a la comunicación es el derecho a recibir información diversa, plural y de calidad que también nos asiste, y es un derecho colectivo".(2016:62)

Frente al desafío de encontrar las ideas y formulaciones organizativas que permitan articular proyectos político-académicos que contribuyan a la transformación social (Tommasino y Cano, 2016) nos interrogamos: ¿Cómo contribuimos desde nuestros espacios y roles a garantizar que los estudiantes universitarios y secundarios ejerzanefectivamente el derecho a la educación? ¿Cómo nos apropiamos y practicamos el derecho a la comunicación?

\section{2. "Curricularizar la extensión" a partir de la producción de contenidos periodísticos}

El corrector del procesador de textos desconoce la palabra "curricularizar". Su mención aparecerá inevitablemente con marcador rojo. ¿Qué significa "curricularizar" la extensión o, como dicen otros, realizar prácticas integrales?

La Universidad de Campinas en Brasil y de la República en Uruguay han venido trabajando en esta línea que, en los hechos, supone incorporar a la formación académica la relación y trabajo con otros actores sociales. La UNER retomó la perspectiva que —especialmente en el período 2006-2014-venía llevando adelante la Universidad de la República en Uruguay (UdelaR) y propuso implementarla con los equipos de cátedra de las Facultades que quisieran sumarse. Esto sucedió en 2014 y 2015.
En nuestro caso, la incorporación al proyecto de curricularización demandó repensar la dinámica de trabajo. El Taller de Producción Periodística es una materia anual incluida en el último año de la Orientación en Periodismo de la Licenciatura en Comunicación Social que se dicta en la Facultad de Ciencias de la Educación en la Universidad Nacional de Entre Ríos (UNER). El objetivo es que los estudiantes universitarios asuman la responsabilidad de comunicar a través de los distintos lenguajes que proponen los medios escritos, digitales, radiales y televisivos. Para ello se desarrolla un recorrido que a través de clases con dinámica de taller y trabajos prácticos vincula lecturas y distintos géneros y subgéneros. Al introducir la extensión al desarrollo del espacio curricular reorganizamos el Programa de Estudios, incorporamos una Unidad que habla de la extensión en la universidad y modificamos parte de la organización de las clases para introducir otras actividades y propuestas de trabajo que prevén horarios extracurriculares. También introdujimos otros modos de evaluar en los que están incluidas las prácticas de extensión.

Hasta antes de sumarnos a este proyecto, la extensión en nuestro equipo de cátedra iba por un carril y el desarrollo de las clases por otro. Si bien invitábamos a los estudiantes universitarios a sumarse a las actividades que hacíamos con escuelas secundarias, esto era independiente de lo que abordábamos en la cátedra.

Lo cierto es que la llegada de la "curricularización de la extensión" a la cátedra supuso más que una palabra difícil de pronunciar: exigió más trabajo para coordinar a los distintos actores y una participación activa del equipo docente. A la vez, trajo consigo una modalidad de enseñanza aprendizaje que ya hicimos nuestra y que, creemos, contribuye a fortalecer los procesos de formación entre los distintos actores involucrados en el sistema educativo.

\section{Universitarios y secundarios hacen periodismo}

Quizás los nombres podrían resumir lo que hacemos. Tal vez podríamos decir que vamos de Parte del Aire al Mal de Ojos y de allí a Va con Onda. El orden en que se los menciona no obedece a otra lógica más que la cronología con que estas producciones periodísticas van realizándose a lo largo del año.

En mayo comienza el ciclo radial que realizan estudiantes universitarios en la radio de la UNER, FM 100.3 de Paraná. Los estudiantes universitarios producen un programa semanal en la radio de la UNER que se emite al aire. Parte del Aire, tal el nombre del magazine radial, contiene un boletín informativo; informes periodísticos; entrevistas; producciones artísticas y agenda cultural. Este trabajo se realiza en grupos de estudiantes que van trabajando de manera articulada. Cada programa se escucha luego en clases y se realizan devoluciones.

En junio, cerca del cierre del primer cuatrimestre, comienza la investigación para el informe periodístico escrito que se presenta en agosto. Luego una selección de estas producciones aparecerá 
en la revista Mal de Ojos. ${ }^{1}$ Ésta reúne una selección de informes periodísticos realizados por los estudiantes a partir de un eje que propone el equipo docente y que tiene como protagonistas a los jóvenes de Entre Ríos y Santa Fe.

En una primera etapa, el trabajo periodístico consiste en investigar una temática acudiendo a lecturas y consultas de bibliografía, documentos, observación y entrevistas, que tienen como fin contribuir a que los estudiantes conozcan el tema en profundidad. Tras finalizar el trabajo de recolección de información llega la etapa de escritura del informe periodístico. Tras ello vendrá la corrección y, luego de una primera devolución del equipo docente, un nuevo ajuste. En una etapa posterior que se realiza entre el equipo docente y los estudiantes se proponen algunos de los informes para ser publicados en la revista.

Después algunos estudiantes compaginan la revista hasta llegar a su impresión y divulgación.

En 2015 la publicación —que empezó participando de las primeras propuestas de extensión- llegó a su décimo número. El tema central fue "Los jóvenes y las relaciones violentas que se producen a partir de las diferencias entre género". Los informes reflejaron problemáticas de jóvenes de Santa Fe y de Entre Ríos ya que los estudiantes de comunicación de UNER investigan estos temas en sus lugares de pertenencia con la intención de aportar contenido de diferentes regiones.

La revista, de circulación gratuita, se distribuye en organizaciones intermedias y medios de comunicación pero fundamentalmente llega a las escuelas secundarias con el fin de que los estudiantes y profesores puedan trabajar en clases algunas de las temáticas que propone. Muchos se ven reflejados en los informes ya que los ejes suelen ser cercanos.

Suele ser recurrente la dificultad para muchos estudiantes de realizar trabajos —en este caso piezas periodísticas- situándose en las exigencias reales que éstas demandan y no en una ficción cuyo destinatario final es el equipo de cátedra. Al proponer que sus producciones escritas circulen en una revista que elaboramos desde extensión y que se lee en organizaciones sociales, escuelas y medios de comunicación, el "escribo como si fuera verdad" se transforma. Producir con la posibilidad de que ese trabajo periodístico se plasme en una publicación que circulará en el espacio social suele generar otra responsabilidad y compromiso al momento de involucrarse con las propuestas de trabajo pero, además, permite participar del espacio público al proponer contenidos periodísticos que contribuyen a introducir otras temáticas desde perspectivas que intentan ser novedosas, creativas e invitar a pensar.

Finalmente, la tercer actividad de curricularización es el ciclo radial Va con Onda, que reúne en un trabajo común a estudiantes y docentes universitarios y secundarios.

\section{La secundaria hace radio}

Dentro de las propuestas de extensión, el desarrollo del ciclo radial Va con Onda, que se emite en la radio de la UNER, es posiblemente la que más articulación demanda entre diferentes actores e instituciones educativas.

Estudiantes secundarios de distintas escuelas públicas de Paraná realizan un magazine de una hora en el que abordan temáticas que definen entre ellos y sus profesores pero que tienen como objetivo plantear temas de interés de la comunidad a la que pertenecen. Los acompañan en la co-conducción los estudiantes de comunicación social que integran el proyecto de curricularización mientras que otros ayudan en la difusión a través de gacetillas y crónicas, en la edición de algunas producciones y en los encuentros de trabajo entre los distintos participantes del programa.

Las escuelas con las que trabajamos cuentan en general con proyectos de radio que se enmarcan en espacios de Formato Complementario o son Bachilleratos con Orientación en Comunicación.

La experiencia nos muestra que estas instancias colocan a los estudiantes universitarios en un rol que les demanda compartir y ejercitar "su saber" con sus pares de secundaria. A la vez, les permite conocerlos mejor y dialogar con ellos sobre algunos aspectos que deben investigar en los informes periodísticos. Durante los meses en que esta actividad se realiza (septiembre y octubre) los que integramos el equipo docente trabajamos como coordinadores para que no haya superposición de temas, distribuimos los bloques por escuelas y garantizamos el contenido. La trama logra armarse con el apoyo de distintos actores que contribuyen a que, finalmente, el programa salga al aire. Directivos de cada escuela; profesores que se comprometen con la propuesta y la llevan adelante (en su mayoría comunicadores sociales); estudiantes que concurren a contra turno; operadores técnicos que editan; representantes de la radio de la UNER que brindan un lugar en la grilla de la programación son, entre otros, actores fundamentales que permiten que el cartel de "Aire" se encienda y eche a andar. 


\section{4}

la llegada de la "curricularización de la

extensión” exigió más trabajo para coordinar

a los distintos actores y una participación

activa del equipo docente

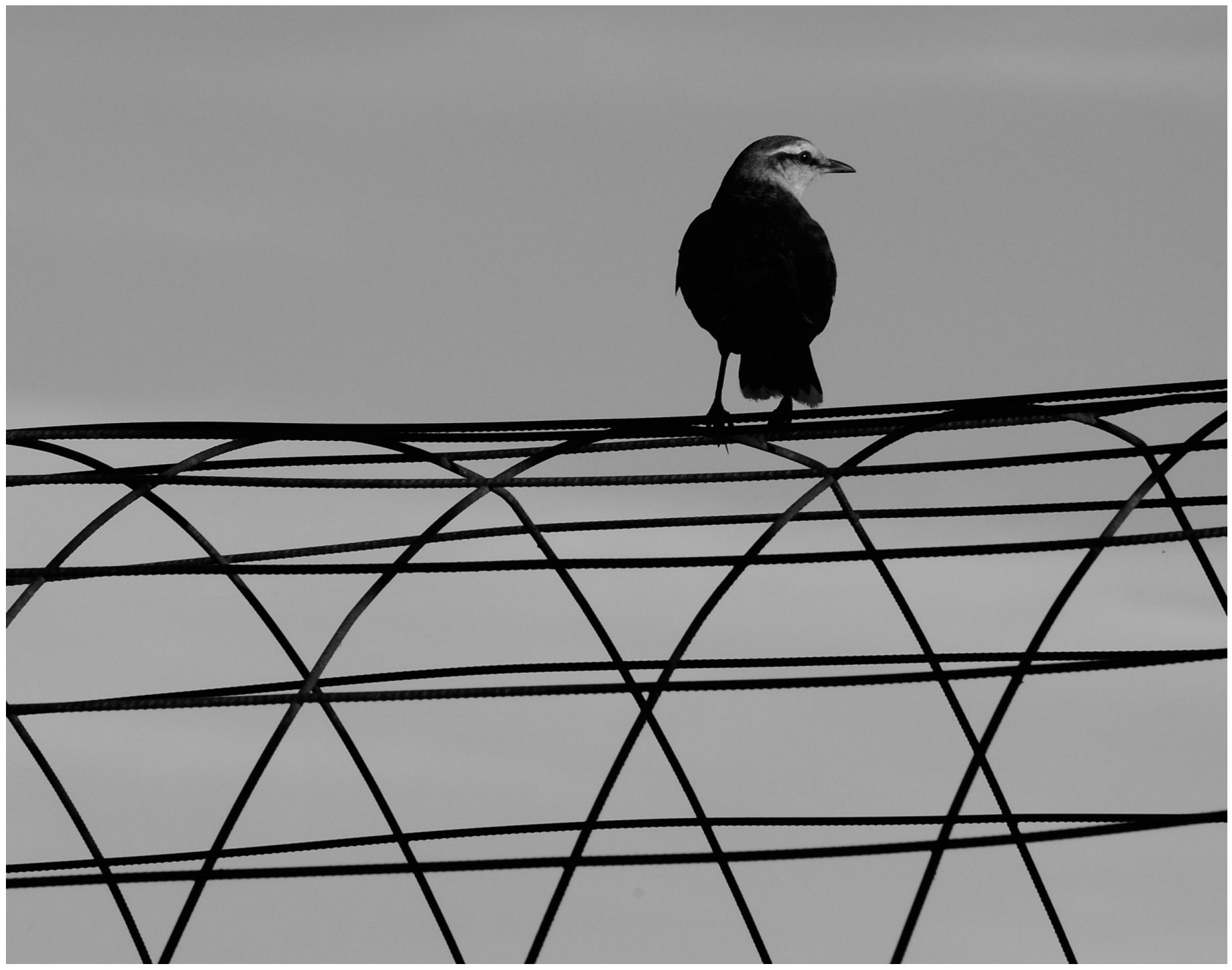

(C) Laura Hormaeche 
En cada emisión se escuchan docentes que dialogan sobre un hecho histórico o sobre la ciencia y las matemáticas en la vida cotidiana; pasan vecinos que cuentan sobre el barrio en donde está una de las escuelas o una madre comparte la receta de las tortas fritas en un día que tocó lluvia. Un ginecólogo explica qué significa la Ley de Educación Sexual Integral y los derechos a acceder a información y a métodos anticonceptivos; una psicóloga analiza por qué hay adolescentes que tienen problemas y escapan de su casa. Además, entre bloque y bloque, los integrantes del programa de radio seleccionan la música para compartir y, en otros, se animan a improvisar un rap sobre la actualidad. Hay eso y hay más. La escuela toma la palabra en la radio para mostrar algo de sus conflictos, de sus trabajos, de la diversidad y heterogeneidad. A pesar de que un ciclo de radio tensiona el formato escolar puesto que obliga a trabajar en horarios que cambian los 80 minutos de cada módulo, la propuesta logra plasmarse en tiempos que transcurren en otros espacios y con otros actores.

A lo largo de los programas se genera una retroalimentación entre los participantes. Los estudiantes secundarios se acercan a la universidad y sienten que pueden ser parte de ella. Sus pares universitarios reconocen lo que saben como parte de su formación universitaria e intentan compartir sus conocimientos.

En las devoluciones que realizan, los alumnos del Taller de Producción Periodística valoran de modo positivo estas instancias de "aprender haciendo con otros" (ver Cuadro 1).

\section{Cuadro 1}

Entrevista a estudiantes de la Escuela Dr. Mariano Moreno, de Paraná, realizada por alumnos del Taller de Producción Periodística en septiembre de $\mathbf{2 0 1 5}$ al finalizar uno de los programas

- ¿En qué consistió la participación de ustedes en el programa de radio de hoy? - Trajimos material creado por nosotros en la escuela y lo presentamos. La idea era preguntarnos que determina que un libro sea un clásico. Charlamos y dimos nuestra conclusión. Contamos lo que sentimos nosotros en base a lo que leímos y hablamos en clases.

- Al principio el tema empezó como una especie de joda. Entrevistábamos a nuestros profesores y nos copó la cara de alegría que ponían cuando decían qué libros les había gustado.

- ¿Qué se les complicó?

— Se nos complicó la redacción. La profesora nos dijo que había que escribir. Costó pero fue lindo. Fue muy buena la experiencia.

Los profesores, que también transpiramos en los antes y durante de cada programa, compartimos el entusiasmo del hacer colectivo y aprendemos otros modos de enseñar a hacer periodismo y comunicarnos. En cada instancia, los contenidos ponen en juego las distintas maneras de comunicar y las diferencias entre medios masivos comerciales y de interés público.

\section{Por dónde andamos}

En el andar que empezamos con las primeras propuestas de extensión en 2008 hemos ido recorriendo un camino de aprendizajes recíprocos entre quienes integramos el equipo, los diferentes participantes, los otros proyectos de extensión y los representantes de la Secretaría de Extensión de la Facultad y de la Universidad. A fines de 2015, la Secretaría de Extensión de la UNER convocó a integrar las funciones de extensión e investigación. En eso estamos; y en el período 2016-2017 la propuesta es continuar desarrollando actividades conjuntas entre estudiantes y docentes de la Universidad y las escuelas públicas secundarias pero introduciendo la investigación.

Las experiencias que venimos desarrollando nos alientan a continuar con esta perspectiva de trabajo en la que situamos a la extensión como proceso necesario del aprendizaje universitario.

En esta etapa decidimos investigar los aprendizajes que promueven en los estudiantes universitarios y secundarios, así como en los docentes, estas propuestas de extensión. Creemos que estos procesos de trabajo entre ambos niveles y actores del sistema educativo contribuyen a generar un espacio de aprendizaje dialógico que, en la posibilidad de vincularse fuera del espacio áulico con funciones en las que docentes y estudiantes trabajamos a la par, favorece otro tipo de relaciones, habilita el reconocimiento de sus identidades, consumos culturales y ayuda a consolidar una formación integral.

Comenzamos planteando qué significa considerar a la educación y a la comunicación como un derecho. Hicimos referencia a la responsabilidad que supone como educadores buscar estrategias para que los jóvenes - no importa el sector social al que pertenecen- reciban educación de calidad y se sientan parte de la Universidad. En este hacer y reflexionar estamos.

\section{Referencias bibliográficas}

Rinesi, E. (2015). Filosofía (y) política de la Universidad. Buenos Aires: Ediciones Universidad Nacional de General Sarmiento.

Tommasino H. y Cano A. (2016). Modelos de extensión universitaria en las universidades latinoamericanas en el siglo XXI: tendencias y controversias. Universidades, (67), 7-24. 